\title{
Review Article \\ On Finsler Geometry and Applications in Mechanics: Review and New Perspectives
}

\author{
J. D. Clayton ${ }^{1,2}$ \\ ${ }^{1}$ Impact Physics, US ARL, Aberdeen, MD 21005-5066, USA \\ ${ }^{2}$ A. James Clark School of Engineering (Adjunct Faculty), University of Maryland, College Park, MD 20742, USA \\ Correspondence should be addressed to J. D. Clayton; john.d.clayton1.civ@mail.mil
}

Received 21 November 2014; Accepted 18 January 2015

Academic Editor: Mahouton N. Hounkonnou

Copyright (c) 2015 J. D. Clayton. This is an open access article distributed under the Creative Commons Attribution License, which permits unrestricted use, distribution, and reproduction in any medium, provided the original work is properly cited.

\begin{abstract}
In Finsler geometry, each point of a base manifold can be endowed with coordinates describing its position as well as a set of one or more vectors describing directions, for example. The associated metric tensor may generally depend on direction as well as position, and a number of connections emerge associated with various covariant derivatives involving affine and nonlinear coefficients. Finsler geometry encompasses Riemannian, Euclidean, and Minkowskian geometries as special cases, and thus it affords great generality for describing a number of phenomena in physics. Here, descriptions of finite deformation of continuous media are of primary focus. After a review of necessary mathematical definitions and derivations, prior work involving application of Finsler geometry in continuum mechanics of solids is reviewed. A new theoretical description of continua with microstructure is then outlined, merging concepts from Finsler geometry and phase field theories of materials science.
\end{abstract}

\section{Introduction}

Mechanical behavior of homogeneous isotropic elastic solids can be described by constitutive models that depend only on local deformation, for example, some metric or strain tensor that may generally vary with position in a body. Materials with microstructure require more elaborate constitutive models, for example, describing lattice orientation in anisotropic crystals, dislocation mechanisms in elastic-plastic crystals, or cracks or voids in damaged brittle or ductile solids. In conventional continuum mechanics approaches, such models typically assign one or more time- and positiondependent vector(s) or higher-order tensor(s), in addition to total deformation or strain, that describe physical mechanisms associated with evolving internal structure.

Mathematically, in classical continuum physics [1-3], geometric field variables describing behavior of a simply connected region of a body depend fundamentally only on referential and spatial coordinate charts $\left\{X^{A}\right\}$ and $\left\{x^{a}\right\}(A, a=$ $1,2, \ldots n)$ related by a diffeomorphism $x=\varphi(X, t)$, with $x$ and
$X$ denoting corresponding points on the spatial and material manifolds covered by corresponding chart(s) and $t$ denoting time. State variables entering response functions depend ultimately only on material points and relative changes in their position (e.g., deformation gradients of first order and possibly higher orders for strain gradient-type models [4]). Geometric objects such as metric tensors, connection coefficients, curvature tensors, and anholonomic objects [5] also depend ultimately only on position. This is true in conventional nonlinear elasticity and plasticity theories $[1,6]$, as well as geometric theories incorporating torsion and/or curvature tensors associated with crystal defects, for example [7-15]. In these classical theories, the metric tensor is always Riemannian (i.e., essentially dependent only upon $x$ or $X$ in the spatial or material setting), meaning the length of a differential line element depends only on position; however, torsion, curvature, and/or covariant derivatives of the metric need not always vanish if the material contains various kinds of defects (non-Euclidean geometry). Connections are linear (i.e., affine). Gauge field descriptions in the context of 
Riemannian metrics and affine connections include [16, 17]. Relevant references in geometry and mathematical physics include [18-26], in addition to those already mentioned. Finite deformation director theories of micropolar type are addressed in the context of Riemannian (as opposed to Finslerian) metrics in $[1,27]$.

Finsler geometry, first attributed to Finsler in 1918 [28], is more general than Riemannian geometry in the sense that the fundamental (metric) tensor generally may depend on additional independent variables, labeled here as $y$ and $Y$ in spatial and material configurations, with corresponding generalized coordinates $\left\{y^{a}\right\}$ and $\left\{Y^{A}\right\}$. Formal definitions will be given later in this paper; for the present immediate discussion, it suffices to mention that each point can be considered endowed with additional degrees-of-freedom beyond $x$ or $X$ and that transformation laws among coordinates as well as connection coefficients (i.e., covariant differentials) generally depend on $y$ or $Y$ as well as $x$ or $X$. Relevant references in mathematics include [29-32]. For descriptions of mechanics of solids, additional degrees-of-freedom can be associated with evolving features of the microstructure of the material, though more general physical interpretations are possible.

The use of Finsler geometry to describe continuum mechanical behavior of solids was perhaps first noted by Kröner in 1968 [33] and Eringen in 1971 [3], the latter reference incorporating some basic identities and definitions derived primarily by Cartan [34], though neither developed a Finsler-based framework more specifically directed towards mechanics of continua. The first theory of Finsler geometry applied to continuum mechanics of solids with microstructure appears to be the purely kinematic theory of Ikeda [35], in a generalization of Cosserat-type kinematics whereby additional degrees-of-freedom are director vectors linked to structure. This theory was essentially extended by Bejancu [30] to distinguish among horizontal and vertical distributions of the fiber bundle of a deforming pseudoFinslerian total space. More complete theories incorporating a Lagrangian functional (leading to physical balance or conservation laws) and couched in terms of Finsler geometry were developed by Saczuk, Stumpf, and colleagues for describing solids undergoing inelastic deformation mechanisms associated with plasticity and/or damage [36-40]. To the author's knowledge, solution of a boundary value problem in solid mechanics using Finsler geometric theory has only been reported once, in [38]. Finsler geometry has been analogously used to generalize fundamental descriptions in other disciplines of physics such as electromagnetism, quantum theory, and gravitation [30, 41-43].

This paper is organized as follows. In Section 2, requisite mathematical background on Finsler geometry (sometimes called Riemann-Finsler geometry [31]) is summarized. In Section 3, the aforementioned theories from continuum physics of solids [30, 35-38, 40] are reviewed and compared. In Section 4, aspects of a new theory, with a primary intention of description of structural transformation processes in real materials, are proposed and evaluated. Conclusions follow in Section 5.

\section{Finsler Geometry: Background}

Notation used in the present section applies to a referential description, that is, the initial state; analogous formulae apply for a spatial description, that is, a deformed body.

2.1. Coordinates and Fundamentals. Denote by $M$ an $n$ dimensional $C^{\infty}$ manifold. Each element (of support) of $M$ is of the form $(X, Y)$, where $X \in M$ and $Y \in T M$, with $T M$ the tangent bundle of $M$. A Finsler structure of $M$ is a function $L: T M \rightarrow[0, \infty)$ with the following three properties [31]:

(i) The fundamental function $L$ is $C^{\infty}$ on $T M \backslash 0$;

(ii) $L(X, \lambda Y)=\lambda L(X, Y) \forall \lambda>0$ (i.e., $L$ is homogeneous of degree one in $Y)$;

(iii) the fundamental tensor $G_{A B}=(1 / 2) \partial^{2}\left(L^{2}\right) / \partial Y^{A} \partial Y^{B}$ is positive definite at every point of $T M \backslash 0$.

Restriction of $L$ to a particular tangent space $T_{X} M$ gives rise to a (local) Minkowski norm

$$
L^{2}(Y)=G_{A B}(Y) Y^{A} Y^{B},
$$

which follows from Euler's theorem and the identity

$$
G_{A B}=\frac{L \partial^{2} L}{\partial Y^{A} \partial Y^{B}}+\left(\frac{\partial L}{\partial Y^{A}}\right)\left(\frac{\partial L}{\partial Y^{B}}\right)
$$

Specifically letting $Y^{A} \rightarrow \mathrm{d} X^{A}$, the length of a differential line element at $X$ depends in general on both $X$ and $Y$ as

$$
|\mathrm{d} \mathbf{X}(X, Y)|=\sqrt{\mathrm{d} \mathbf{X} \cdot \mathrm{d} \mathbf{X}}=\left[G_{A B}(X, Y) \mathrm{d} X^{A} \mathrm{~d} X^{B}\right]^{1 / 2}
$$

A Finsler manifold $(M, F)$ reduces to a Minkowskian manifold when $L$ does not depend on $X$ and to a Riemannian manifold when $L$ does not depend on $Y$. In the latter case, a Riemannian metric tensor is $G_{A B}(X) d X^{A} \otimes d X^{B}$. Cartan's tensor, with the following fully symmetric covariant components, is defined for use later:

$$
C_{A B C}=\frac{1}{2} \frac{\partial G_{A B}}{\partial Y^{C}}=\frac{1}{4} \frac{\partial^{3}\left(L^{2}\right)}{\partial Y^{A} \partial Y^{B} \partial Y^{C}} .
$$

Consider now a coordinate transformation to another chart on $M$; for example,

$$
\widetilde{X}^{A}=\widetilde{X}^{A}\left(X^{1}, X^{2}, \ldots, X^{n}\right), \quad \widetilde{Y}^{A}=\left(\frac{\partial \widetilde{X}^{A}}{\partial X^{B}}\right) Y^{B} .
$$

From the chain rule, holonomic basis vectors on TM then transform as $[30,31]$

$$
\begin{gathered}
\frac{\partial}{\partial \widetilde{X}^{A}}=\frac{\partial X^{B}}{\partial \widetilde{X}^{A}} \frac{\partial}{\partial X^{B}}+\frac{\partial^{2} X^{B}}{\partial \widetilde{X}^{A} \partial \widetilde{X}^{C}} \widetilde{Y}^{C} \frac{\partial}{\partial Y^{B}}, \\
\frac{\partial}{\partial \widetilde{Y}^{A}}=\frac{\partial X^{B}}{\partial \widetilde{X}^{A}} \frac{\partial}{\partial Y^{B}} .
\end{gathered}
$$


2.2. Connections and Differentiation. Christoffel symbols of the second kind derived from the symmetric fundamental tensor are

$$
\gamma_{B C}^{A}=\frac{1}{2} G^{A D}\left(\frac{\partial G_{B D}}{\partial X^{C}}+\frac{\partial G_{C D}}{\partial X^{B}}-\frac{\partial G_{B C}}{\partial X^{D}}\right) .
$$

Lowering and raising of indices are enabled via $G_{A B}$ and its inverse $G^{A B}$. Nonlinear connection coefficients on $T M \backslash 0$ are defined as

$$
N_{B}^{A}=\gamma_{B C}^{A} Y^{C}-C_{B C}^{A} \gamma_{D E}^{C} Y^{D} Y^{E}=\frac{1}{2} \frac{\partial G^{A}}{\partial Y^{B}},
$$

where $G^{A}=\gamma_{B C}^{A} Y^{B} Y^{C}$. The following nonholonomic bases are then introduced:

$$
\frac{\delta}{\delta X^{A}}=\frac{\partial}{\partial X^{A}}-N_{A}^{B} \frac{\partial}{\partial Y^{B}}, \quad \delta Y^{A}=d Y^{A}+N_{B}^{A} d X^{B} .
$$

It can be shown that unlike (6), these nonholonomic bases obey simple transformation laws like (7). The set $\left\{\delta / \delta X^{A}, \partial / \partial Y^{A}\right\}$ serves as a convenient local basis for $T(T M \backslash$ $0)$; its dual set $\left\{d X^{A}, \delta Y^{A}\right\}$ applies for the cotangent bundle $T^{*}(T M \backslash 0)$. A natural Riemannian metric can then be introduced, called a Sasaki metric [31]:

$$
\mathbf{G}(X, Y)=G_{A B} d X^{A} \otimes d X^{B}+G_{A B} \delta Y^{A} \otimes \delta Y^{B} .
$$

The horizontal subspace spanned by $\left\{\delta / \delta X^{A}\right\}$ is orthogonal to the vertical subspace spanned by $\left\{\partial / \partial Y^{B}\right\}$ with respect to this metric. Covariant derivative $\nabla$, or collectively connection 1-forms $\omega_{B}^{A}$, define a linear connection on pulled-back bundle $\pi^{*} T M$ over $T M \backslash 0$. Letting $v$ denote an arbitrary direction,

$$
\nabla_{v} \frac{\partial}{\partial X^{A}}=\omega_{A}^{B}(v) \frac{\partial}{\partial X^{B}}, \quad \nabla_{v} d X^{A}=-\omega_{B}^{A}(v) d X^{B}
$$

A number of linear connections have been introduced in the Finsler literature $[30,31]$. The Chern-Rund connection $[29,44]$ is used most frequently in applications related to the present paper. It is a unique linear connection on $\pi^{*} T M$ characterized by the structural equations [31]

$$
\begin{gathered}
d\left(d X^{A}\right)-d X^{B} \wedge \omega_{B}^{A}=0, \\
d G_{A B}-G_{B C} \omega_{A}^{C}-G_{A C} \omega_{B}^{C}=2 C_{A B C} \delta Y^{C} .
\end{gathered}
$$

The first structure equation implies torsion freeness and results in

$$
\omega_{B}^{A}=\Gamma_{C B}^{A} d X^{C}, \quad \Gamma_{B C}^{A}=\Gamma_{C B}^{A} .
$$

The second leads to the connection coefficients

$$
\Gamma_{B C}^{A}=\frac{1}{2} G^{A D}\left(\frac{\delta G_{B D}}{\delta X^{C}}+\frac{\delta G_{C D}}{\delta X^{B}}-\frac{\delta G_{B C}}{\delta X^{D}}\right) .
$$

When a Finsler manifold degenerates to a Riemannian manifold, $N_{B}^{A}=0$ and $\Gamma_{B C}^{A}=\gamma_{B C}^{A}$. Cartan's connection 1-forms are defined by $\omega_{B}^{A}+C_{D B}^{A} \delta Y^{D}$ where $\omega_{B}^{A}$ correspond to (14); its coordinate formulae and properties are listed in [3]. It has been shown [45] how components of Cartan's connection on a Finsler manifold can be obtained as the induced connection of an enveloping space (with torsion) of dimension $2 n$. When a Finsler manifold degenerates to a locally Minkowski space ( $L$ independent of $X$ ), then $\Gamma_{B C}^{A}=\gamma_{B C}^{A}=0$. Gradients of bases with respect to the Chern-Rund connection and Cartan tensor are

$$
\nabla_{\delta / \delta X^{A}} \frac{\delta}{\delta X^{B}}=\Gamma_{A B}^{C} \frac{\delta}{\delta X^{C}}, \quad \nabla_{\delta / \delta X^{A}} \frac{\partial}{\partial Y^{B}}=C_{A B}^{C} \frac{\partial}{\partial Y^{C}} .
$$

As an example of covariant differentiation on a Finsler manifold with Chern-Rund connection $\nabla$, consider a $\left(\begin{array}{l}1 \\ 1\end{array}\right)$ tensor field $\mathbf{T}=T_{B}^{A}\left(\partial / \partial X^{A}\right) \otimes d X^{B}$ on the manifold $T M \backslash 0$. The covariant differential of $\mathbf{T}(X, Y)$ is

$$
\begin{aligned}
(\nabla T)_{B}^{A} & =d T_{B}^{A}+T_{B}^{C} \omega_{C}^{A}-T_{C}^{A} \omega_{B}^{C} \\
& =T_{B \mid C}^{A} d X^{C}+T_{B \| C}^{A} \delta Y^{C} \\
& =\left(\nabla_{\delta / \delta X^{C}} T\right)_{B}^{A} d X^{C}+\left(\nabla_{\partial / \partial Y^{C} T}\right)_{B}^{A} \delta Y^{C} \\
& =\left(\frac{\delta T_{B}^{A}}{\delta X^{C}}+T_{B}^{D} \Gamma_{C D}^{A}-T_{D}^{A} \Gamma_{C B}^{D}\right) d X^{C}+\left(\frac{\partial T_{B}^{A}}{\partial Y^{C}}\right) \delta Y^{C}
\end{aligned}
$$

Notations $(\cdot)_{\mid A}$ and $(\cdot)_{\| A}$ denote respective horizontal and vertical covariant derivatives with respect to $\nabla$.

2.3. Geometric Quantities and Identities. Focusing again on the Chern-Rund connection $\nabla$, curvature 2-forms are

$$
\begin{aligned}
\Omega_{B}^{A}= & d\left(\omega_{B}^{A}\right)-\omega_{B}^{C} \wedge \omega_{C}^{A} \\
= & \frac{1}{2} R_{B C D}^{A} d X^{C} \wedge d X^{D}+P_{B C D}^{A} d X^{C} \wedge \delta Y^{D} \\
& +\frac{1}{2} Q_{B C D}^{A} \delta Y^{C} \wedge \delta Y^{D},
\end{aligned}
$$

with $d(\cdot)$ the exterior derivative and $\wedge$ the wedge product (no factor of 1/2). HH-, HV-, and VV-curvature tensors of the Chern-Rund connection have respective components

$$
\begin{aligned}
& R_{B C D}^{A}=\frac{\delta \Gamma_{B D}^{A}}{\delta X^{C}}-\frac{\delta \Gamma_{B C}^{A}}{\delta X^{D}}+\Gamma_{E C}^{A} \Gamma_{B D}^{E}-\Gamma_{E D}^{A} \Gamma_{B C}^{E}, \\
& P_{B C D}^{A}=-\frac{\partial \Gamma_{B C}^{A}}{\partial Y^{D}}, \quad Q_{B C D}^{A}=0 .
\end{aligned}
$$

VV-curvature vanishes, $\mathrm{HV}$-curvature obeys $P_{B C D}^{A}=P_{C B D}^{A}$, and a Bianchi identity for $\mathrm{HH}$-curvature is

$$
R_{B C D}^{A}+R_{C D B}^{A}+R_{D B C}^{A}=0 .
$$

When a Finsler manifold degenerates to a Riemannian manifold, then $R_{B C D}^{A}$ become the components of the usual curvature tensor of Riemannian geometry constructed from $\gamma_{B C}^{A}$, and $P_{B C D}^{A}=0$. All curvatures vanish in locally Minkowski spaces. It is not always possible to embed a Finsler 
space in a Riemannian space without torsion, but it is possible to determine the metric and torsion tensors of a space of dimension $2 n-1$ in such a way that any $n$-dimensional Finsler space is a nonholonomic subspace of such a space with torsion [46].

Nonholonomicity (i.e., nonintegrability) of the horizontal distribution is measured by [32]

$$
\left[\frac{\delta}{\delta X^{A}}, \frac{\delta}{\delta X^{B}}\right]=\left(\frac{\delta N_{A}^{C}}{\delta X^{B}}-\frac{\delta N_{B}^{C}}{\delta X^{A}}\right) \frac{\partial}{\partial Y^{C}}=\Lambda_{A B}^{C} \frac{\partial}{\partial Y^{C}}
$$

where $[\cdot, \cdot]$ is the Lie bracket and $\Lambda_{A B}^{C}$ can be interpreted as components of a torsion tensor [30]. For the Chern-Rund connection [31],

$$
\Lambda_{A B}^{C}=-R_{D A B}^{C} Y^{D} .
$$

Since Lie bracket (21) is strictly vertical, the horizontal distribution spanned by $\left\{\delta / \delta X^{A}\right\}$ is not involutive [31].

\section{Applications in Solid Mechanics: 1973-2003}

3.1. Early Director Theory. The first application of Finsler geometry to finite deformation continuum mechanics is credited to Ikeda [35], who developed a director theory in the context of (pseudo-) Finslerian manifolds. A slightly earlier work [47] considered a generalized space (not necessarily Finslerian) comprised of finitely deforming physical and geometrical fields. Paper [35] is focused on kinematics and geometry: descriptions of deformations of the continuum and the director vector fields and their possible interactions, metric tensors (i.e., fundamental tensors), and gradients of motions. Covariant differentials are defined that can be used in field theories of Finsler space [41, 48]. Essential concepts from [35] are reviewed and analyzed next.

Let $M$ denote a pseudo-Finsler manifold in the sense of [35], representative of a material body with microstructure. Let $X \in M$ denote a material point with coordinate chart $\left\{X^{A}\right\},(A=1,2,3)$ covering the body in its undeformed state. A set of director vectors $\mathbf{D}_{(\alpha)}$ is attached to each $X$, where $(\alpha=1,2, \ldots, p)$. In component form, directors are written $\left\{D_{(\alpha)}^{A}\right\}$. In the context of notation in Section 2, $M$ is similar to a Finsler manifold with $Y^{A} \rightarrow D_{(\alpha)}^{A}$, though the director theory involves more degrees-of-freedom when $p>1$, and no fundamental function is necessarily introduced. Let $x$ denote the spatial location in a deformed body of a point initially at $X$, and let $\mathbf{d}_{(\alpha)}$ denote a deformed director vector. The deformation process is described by $(a=1,2,3)$

$$
x^{a}=x^{a}\left(X^{A}\right), \quad d_{(\alpha)}^{a}=d_{(\alpha)}^{a}\left[D_{(\beta)}^{A}\left(X^{B}\right)\right]=d_{(\alpha)}^{a}\left(X^{B}\right) .
$$

The deformation gradient and its inverse are

$$
F_{A}^{a}=\frac{\partial x^{a}}{\partial X^{A}}, \quad F_{a}^{-1 A}=\frac{\partial X^{A}}{\partial x^{a}} .
$$

The following decoupled transformations are posited:

$$
\mathrm{d} x^{a}=F_{A}^{a} \mathrm{~d} X^{A}, \quad d_{(\alpha)}^{a}=B_{A}^{a} D_{(\alpha)}^{A},
$$

with $B_{A}^{a}=B_{A}^{a}(X)$. Differentiating the second of (25),

$$
\mathrm{d} d_{(\alpha)}^{a}=E_{(\alpha) A}^{a} \mathrm{~d} X^{A}=\left(B_{B: A}^{a} D_{(\alpha)}^{B}+B_{B}^{a} D_{(\alpha): A}^{B}\right) \mathrm{d} X^{A},
$$

where $(\cdot)_{: A}$ denotes the total covariant derivative $[20,26]$. Differential line and director elements can be related by

$$
\mathrm{d} d_{(\alpha)}^{a}=f_{(\alpha) b}^{a} \mathrm{~d} x^{b}=F_{b}^{-1 A} E_{(\alpha) A}^{a} \mathrm{~d} x^{b} .
$$

Let $G_{A B}(X)$ denote the metric in the reference configuration, such that $\mathrm{d} S^{2}=\mathrm{d} X^{A} G_{A B} \mathrm{~d} X^{B}$ is a measure of length $\left(G_{A B}=\delta_{A B}\right.$ for Cartesian coordinates $\left.\left\{X^{A}\right\}\right)$. Fundamental tensors in the spatial frame describing strains of the continuum and directors are

$$
C_{a b}=F_{a}^{-1 A} G_{A B} F_{b}^{-1 B}, \quad C_{a b}^{(\alpha \beta)}=E_{(\alpha) a}^{-1 A} G_{A B} E_{(\alpha) b}^{-1 B} .
$$

Let $\bar{\Gamma}_{B C}^{A}$ and $\bar{\Gamma}_{\beta \gamma}^{\alpha}$ denote coefficients of linear connections associated with continuum and director fields, related by

$$
\bar{\Gamma}_{B C}^{A}=D_{(\alpha)}^{A} D_{B}^{(\beta)} D_{C}^{(\gamma)} \bar{\Gamma}_{\beta \gamma}^{\alpha}+D_{(\alpha)}^{A} D_{C: B}^{(\alpha)}
$$

where $D_{(\alpha)}^{A} D_{B}^{(\alpha)}=\delta_{B}^{A}$ and $D_{(\beta)}^{A} D_{A}^{(\alpha)}=\delta_{\beta}^{\alpha}$. The covariant differential of a referential vector field $\mathbf{V}$, where locally $\mathbf{V}(X) \in T_{X} M$, with respect to this connection is

$$
(\bar{\nabla} V)^{A}=d V^{A}+\bar{\Gamma}_{B C}^{A} V^{C} \mathrm{~d} X^{B}
$$

the covariant differential of a spatial vector field $v$, where locally $\boldsymbol{v}(x) \in T_{x} M$, is defined as

$$
(\bar{\nabla} v)^{a}=d v^{a}+\bar{\Gamma}_{b c}^{a} v^{c} \mathrm{~d} x^{b}+\bar{\Gamma}_{b c}^{(\alpha) a} v^{c} \mathrm{~d} x^{b}
$$

with, for example, the differential of $v^{a}\left(x, \mathbf{d}_{(\alpha)}\right)$ given by $d v^{a}=$ $\left(\partial v^{a} / \partial x^{b}\right) \mathrm{d} x^{b}+\left(\partial v^{a} / \partial d_{(\alpha)}^{b}\right) \mathrm{d} d_{(\alpha)}^{b}$. Euler-Schouten tensors are

$$
H_{b c}^{(\alpha) a}=F_{A}^{a} E_{(\alpha) c: b}^{-1 A}, \quad K_{b c}^{(\alpha) a}=E_{(\alpha) A}^{a} F_{c: b}^{-1 A} .
$$

Ikeda [35] implies that fundamental variables entering a field theory for directed media should include the set (F, B, E, H, K). Given the fields in (23), the kinematicgeometric theory is fully determined once $\bar{\Gamma}_{\beta \gamma}^{\alpha}$ are defined. The latter coefficients can be related to defect content in a crystal. For example, setting $\bar{\Gamma}_{\beta \gamma}^{\alpha}=0$ results in distant parallelism associated with dislocation theory [7], in which case (29) gives the negative of the wryness tensor; more general theory is needed, however, to represent disclination defects [49] or other general sources of incompatibility [6, 50] requiring a nonvanishing curvature tensor. The directors themselves can be related to lattice directions, slip vectors in crystal plasticity $[51,52]$, preferred directions for twinning $[6,53]$, or planes intrinsically prone to cleavage fracture [54], for example. Applications of multifield theory [47] towards multiscale descriptions of crystal plasticity $[50,55]$ are also possible. 
3.2. Kinematics and Gauge Theory on a Fiber Bundle. The second known application of Finsler geometry towards finite deformation of solid bodies appears in Chapter 8 of the book of Bejancu [30]. Content in [30] extends and formalizes the description of Ikeda [35] using concepts of tensor calculus on the fiber bundle of a (generalized pseudo-) Finsler manifold. Geometric quantities appropriate for use in gauge-invariant Lagrangian functions are derived. Relevant features of the theory in [30] are reviewed and analyzed in what follows next.

Define $\zeta=(Z, \pi, M, U)$ as a fiber bundle of total space $Z$, where $\pi: Z \rightarrow M$ is the projection to base manifold $M$ and $U$ is the fiber. Dimensions of $M$ and $U$ are $n(n=3$ for a solid volume) and $p$, respectively; the dimension of $Z$ is $r=n+p$. Coordinates on $Z$ are $\left\{X^{A}, D^{\alpha}\right\}$, where $X \in M$ is a point on the base body in its reference configuration, and $D$ is a director of dimension $p$ that essentially replaces multiple directors of dimension 3 considered in Section 3.1. The natural basis on $Z$ is the field of frames $\left\{\partial / \partial X^{A}, \partial / \partial D^{\alpha}\right\}$. Let $N_{A}^{\alpha}(X, D)$ denote nonlinear connection coefficients on $Z$, and introduce the nonholonomic bases

$$
\frac{\delta}{\delta X^{A}}=\frac{\partial}{\partial X^{A}}-N_{A}^{\alpha} \frac{\partial}{\partial D^{\alpha}}, \quad \delta D^{\alpha}=d D^{\alpha}+N_{A}^{\alpha} d X^{A} .
$$

Unlike $\left\{\partial / \partial X^{A}\right\}$, these nonholonomic bases obey simple transformation laws; $\left\{\delta / \delta X^{A}, \partial / \partial D^{\alpha}\right\}$ serves as a convenient local basis for $T Z$ adapted to a decomposition into horizontal and vertical distributions:

$$
T Z=H Z \oplus V Z
$$

Dual set $\left\{d X^{A}, \delta D^{\alpha}\right\}$ is a local basis on $T^{*} Z$. A fundamental tensor (i.e., metric) for the undeformed state is

$$
\begin{aligned}
\mathbf{G}(X, D)= & G_{A B}(X, D) d X^{A} \otimes d X^{B} \\
& +G_{\alpha \beta}(X, D) \delta D^{\alpha} \otimes \delta D^{\beta}
\end{aligned}
$$

Let $\nabla$ denote covariant differentiation with respect to a connection on the vector bundles $H Z$ and $V Z$, with

$$
\begin{aligned}
\nabla_{\delta / \delta X^{A}} \frac{\delta}{\delta X^{B}}=\Gamma_{A B}^{C} \frac{\delta}{\delta X^{C}}, & \nabla_{\delta / \delta X^{A}} \frac{\partial}{\partial D^{\alpha}}=C_{A \alpha}^{\beta} \frac{\partial}{\partial D^{\beta}} \\
\nabla_{\partial / \partial D^{\alpha}} \frac{\delta}{\delta X^{A}}=c_{\alpha A}^{B} \frac{\delta}{\delta X^{B}}, & \nabla_{\partial / \partial D^{\alpha}} \frac{\partial}{\partial D^{\beta}}=\chi_{\alpha \beta}^{\gamma} \frac{\partial}{\partial D^{\gamma}}
\end{aligned}
$$

Consider a horizontal vector field $\mathbf{V}=V^{A}\left(\delta / \delta X^{A}\right)$ and a vertical vector field $\mathbf{W}=W^{\alpha}\left(\partial / \partial D^{\alpha}\right)$. Horizontal and vertical covariant derivatives are defined as

$$
\begin{aligned}
V_{\mid B}^{A}=\frac{\delta V^{A}}{\delta X^{B}}+\Gamma_{B C}^{A} V^{C}, & W_{\mid B}^{\alpha}=\frac{\delta W^{\alpha}}{\delta X^{B}}+C_{B \beta}^{\alpha} W^{\beta} ; \\
V_{\| \beta}^{A}=\frac{\delta V^{A}}{\delta D^{\beta}}+c_{\beta C}^{A} V^{C}, & W_{\| \beta}^{\alpha}=\frac{\delta W^{\alpha}}{\delta D^{\beta}}+\chi_{\beta \delta}^{\alpha} W^{\delta} .
\end{aligned}
$$

Generalization to higher-order tensor fields is given in [30]. In particular, the following coefficients are assigned to the socalled gauge $\mathrm{H}$-connection on $Z$ :

$$
\begin{gathered}
\Gamma_{B C}^{A}=\frac{1}{2} G^{A D}\left(\frac{\delta G_{B D}}{\delta X^{C}}+\frac{\delta G_{C D}}{\delta X^{B}}-\frac{\delta G_{B C}}{\delta X^{D}}\right), \\
C_{A \beta}^{\alpha}=\frac{\partial N_{A}^{\alpha}}{\partial D^{\beta}}, \quad c_{\alpha B}^{A}=0, \\
\chi_{\beta \gamma}^{\alpha}=\frac{1}{2} G^{\alpha \delta}\left(\frac{\partial G_{\beta \delta}}{\partial D^{\gamma}}+\frac{\partial G_{\gamma \delta}}{\partial D^{\beta}}-\frac{\partial G_{\beta \gamma}}{\partial D^{\delta}}\right) .
\end{gathered}
$$

Comparing with the formal theory of Finsler geometry outlined in Section 2, coefficients $\Gamma_{B C}^{A}$ are analogous to those of the Chern-Rund connection, and (36) is analogous to (16). The generalized pseudo-Finslerian description of [30] reduces to Finsler geometry of [31] when $p=n$ and a fundamental function $L$ exists from which metric tensors and nonlinear connection coefficients can be derived.

Let $Q^{K}(X, D)$ denote a set of differentiable state variables, where $K=1,2, \ldots, r$. A Lagrangian function $\mathbb{Q}$ of the following form is considered on $Z$ :

$$
\mathfrak{L}(X, D)=\mathfrak{L}\left[G_{A B}, G_{\alpha \beta}, Q^{K}, \frac{\delta Q^{K}}{\delta X^{A}}, \frac{\partial Q^{K}}{\partial D^{\alpha}}\right] .
$$

Let $\Omega$ be a compact domain of $Z$, and define the functional (action integral)

$$
\begin{gathered}
I(\Omega)=\int_{\Omega} \widehat{\mathfrak{\Omega}}(X, D) d X^{1} \cdots \wedge d X^{n} \wedge d D^{1} \cdots \wedge d D^{p}, \\
\widehat{\mathbf{\Omega}}=\left[\left|\operatorname{det}\left(G_{A B}\right) \| \operatorname{det}\left(G_{\alpha \beta}\right)\right|\right]^{1 / 2} \mathbf{R} .
\end{gathered}
$$

Euler-Lagrange equations referred to the reference configuration follow from the variational principle $\delta I=0$ :

$$
\frac{\partial \widehat{\mathbb{Q}}}{\partial Q^{K}}=\frac{\partial}{\partial X^{A}}\left[\frac{\partial \widehat{\mathbb{Q}}}{\partial\left(\partial Q^{K} / \partial X^{A}\right)}\right]+\frac{\partial}{\partial D^{\alpha}}\left[\frac{\partial \widehat{\mathbb{Q}}}{\partial\left(\partial Q^{K} / \partial D^{\alpha}\right)}\right] .
$$

These can be rewritten as invariant conservation laws involving horizontal and vertical covariant derivatives with respect to the gauge- $\mathrm{H}$ connection [30].

Let $\zeta^{\prime}=\left(Z^{\prime}, \pi^{\prime}, M^{\prime}, U^{\prime}\right)$ be the deformed image of fiber bundle $\zeta$, representative of deformed geometry of the body, for example. Dimensions of $M^{\prime}$ and $U^{\prime}$ are $n$ and $p$, respectively; the dimension of $Z^{\prime}$ is $r=n+p$. Coordinates on $Z^{\prime}$ are $\left\{x^{a}, d^{\alpha^{\prime}}\right\}$, where $x \in M^{\prime}$ is a point on the base body in its current configuration, and $d$ is a director of dimension $p$. The natural basis on $Z^{\prime}$ is the field of frames $\left\{\partial / \partial x^{a}, \partial / \partial d^{\alpha^{\prime}}\right\}$. Let $N_{a}^{\alpha^{\prime}}(x, d)$ denote nonlinear connection coefficients on $Z^{\prime}$, and introduce the nonholonomic bases

$$
\frac{\delta}{\delta x^{a}}=\frac{\partial}{\partial x^{a}}-N_{a}^{\alpha^{\prime}} \frac{\partial}{\partial d^{\alpha^{\prime}}}, \quad \delta d^{\alpha^{\prime}}=d d^{\alpha^{\prime}}+N_{a}^{\alpha^{\prime}} d x^{a} .
$$

These nonholonomic bases obey simple transformation laws; $\left\{\delta / \delta x^{a}, \partial / \partial d^{\alpha^{\prime}}\right\}$ serves as a convenient local basis for $T Z^{\prime}$ where

$$
T Z^{\prime}=H Z^{\prime} \oplus V Z^{\prime}
$$


Dual set $\left\{d x^{a}, \delta d^{\alpha^{\prime}}\right\}$ is a local basis on $T^{*} Z^{\prime}$. Deformation of $(Z, M)$ to $\left(Z^{\prime}, M^{\prime}\right)$ is dictated by diffeomorphisms in local coordinates:

$$
x^{a}=x^{a}\left(X^{A}\right), \quad d^{\alpha^{\prime}}=B_{\beta}^{\alpha^{\prime}}(X) D^{\beta}
$$

Let the usual (horizontal) deformation gradient and its inverse have components

$$
F_{A}^{a}=\frac{\partial x^{a}}{\partial X^{A}}, \quad F_{a}^{-1 A}=\frac{\partial X^{A}}{\partial x^{a}} .
$$

It follows from (45) that, similar to (6) and (7),

$$
\begin{gathered}
\frac{\partial}{\partial X^{A}}=F_{A}^{a} \frac{\partial}{\partial x^{a}}+\frac{\partial B_{\beta}^{\alpha^{\prime}}}{\partial X^{A}} D^{\beta} \frac{\partial}{\partial d^{\alpha^{\prime}}} \\
\frac{\partial}{\partial D^{\alpha}}=B_{\alpha}^{\beta^{\prime}} \frac{\partial}{\partial d^{\beta^{\prime}}} .
\end{gathered}
$$

Nonlinear connection coefficients on $Z$ and $Z^{\prime}$ can be related by

$$
N_{a}^{\alpha^{\prime}} F_{A}^{a}=N_{A}^{\beta} B_{\beta}^{\alpha^{\prime}}-\left(\frac{\partial B_{\beta}^{\alpha^{\prime}}}{\partial X^{A}}\right) D^{\beta}
$$

Metric tensor components on $Z$ and $Z^{\prime}$ can be related by

$$
G_{a b} F_{A}^{a} F_{B}^{b}=G_{A B}, \quad G_{\alpha^{\prime} \beta^{\prime}} B_{\delta}^{\alpha^{\prime}} B_{\gamma}^{\beta^{\prime}}=G_{\delta \gamma}
$$

Linear connection coefficients on $Z$ and $Z^{\prime}$ can be related by

$$
\begin{gathered}
\Gamma_{b c}^{a} F_{B}^{b} F_{C}^{c}=\Gamma_{B C}^{A} F_{A}^{a}-\frac{\partial F_{C}^{a}}{\partial X^{B}}, \\
C_{a \beta^{\prime}}^{\alpha^{\prime}} F_{A}^{a} B_{\gamma}^{\beta^{\prime}}=C_{A \gamma}^{\mu} B_{\mu}^{\alpha^{\prime}}-\frac{\partial B_{\gamma}^{\alpha^{\prime}}}{\partial X^{A}}, \\
c_{\alpha^{\prime} b}^{a} F_{A}^{b} B_{\beta}^{\alpha^{\prime}}=c_{\beta A}^{B} F_{B}^{a}, \\
\chi_{\beta^{\prime} \delta^{\prime}}^{\alpha^{\prime}} B_{\gamma}^{\beta^{\prime}} B_{\eta}^{\delta^{\prime}}=\chi_{\gamma \eta}^{\varepsilon} B_{\varepsilon}^{\alpha^{\prime}} .
\end{gathered}
$$

Using the above transformations, a complete gauge $\mathrm{H}$ connection can be obtained for $Z^{\prime}$ from reference quantities on $Z$ if the deformation functions in (45) are known. A Lagrangian can then be constructed analogously to (40), and Euler-Lagrange equations for the current configuration of the body can be derived from a variational principle where the action integral is taken over the deformed space. In application of pseudo-Finslerian fiber bundle theory similar to that outlined above, Fu et al. [37] associate $d$ with the director of an oriented area element that may be degraded in strength due to damage processes such as fracture or void growth in the material. See also related work in [39].

3.3. Recent Theories in Damage Mechanics and Finite Plasticity. Saczuk et al. $[36,38,40]$ adapted a generalized version of pseudo-Finsler geometry similar to the fiber bundle approach of [30] and Section 3.2 to describe mechanics of solids with microstructure undergoing finite elastic-plastic or elasticdamage deformations. Key new contributions of these works include definitions of total deformation gradients consisting of horizontal and vertical components and Lagrangian functions with corresponding energy functionals dependent on total deformations and possibly other state variables. Constitutive relations and balance equations are then derived from variations of such functionals. Essential details are compared and analyzed in the following discussion. Notation usually follows that of Section 3.2, with a few generalizations defined as they appear.

Define $\zeta=(Z, \pi, M, U)$ as a fiber bundle of total space $Z$, where $\pi: Z \rightarrow M$ is the projection to base manifold $M$ and $U$ is the fiber. Dimensions of $M$ and $U$ are 3 and $p$, respectively; the dimension of $Z$ is $r=3+p$. Coordinates on $Z$ are $\left\{X^{A}, D^{\alpha}\right\}$, where $X \in M$ is a point on the base body in its reference configuration, and $D$ is a vector of dimension $p$. Let $\zeta^{\prime}=\left(Z^{\prime}, \pi^{\prime}, M^{\prime}, U^{\prime}\right)$ be the deformed image of fiber bundle $\zeta$; dimensions of $M^{\prime}$ and $U^{\prime}$ are 3 and $p$, respectively; the dimension of $Z^{\prime}$ is $r=3+p$. Coordinates on $Z^{\prime}$ are $\left\{x^{a}, d^{\alpha^{\prime}}\right\}$, where $x \in M^{\prime}$ is a point on the base body in its current configuration, and $d$ is a vector of dimension $p$. Tangent bundles can be expressed as direct sums of horizontal and vertical distributions:

$$
T Z=H Z \oplus V Z, \quad T Z^{\prime}=H Z^{\prime} \oplus V Z^{\prime}
$$

Deformation of $Z$ to $Z^{\prime}$ is locally represented by the smooth and invertible coordinate transformations

$$
x^{a}=x^{a}\left(X^{A}, D^{\alpha}\right), \quad d^{\alpha^{\prime}}=B_{\beta}^{\alpha^{\prime}} D^{\beta}
$$

where in general the director deformation map [38]

$$
B_{\beta}^{\alpha^{\prime}}=B_{\beta}^{\alpha^{\prime}}(X, D)
$$

Total deformation gradient $\mathfrak{F}: T Z \rightarrow T Z^{\prime}$ is defined as

$$
\mathfrak{F}=\overline{\mathbf{F}}+\widehat{\mathbf{F}}=x_{\mid A}^{a} \frac{\delta}{\delta x^{a}} \otimes d X^{A}+x_{\| \alpha}^{a} \delta_{a}^{\beta^{\prime}} \frac{\partial}{\partial d^{\beta^{\prime}}} \otimes \delta D^{\alpha}
$$

In component form, its horizontal and vertical parts are

$$
\begin{gathered}
\bar{F}_{A}^{a}=x_{\mid A}^{a}=\frac{\delta x^{a}}{\delta X^{A}}+\Gamma_{A C}^{B} \delta_{c}^{C} \delta_{B}^{a} x^{c} \\
\widehat{F}_{\alpha}^{\beta^{\prime}}=x_{\| \alpha}^{a} \delta_{a}^{\beta^{\prime}}=\left(\frac{\partial x^{a}}{\partial D^{\alpha}}+C_{A C}^{B} \delta_{\alpha}^{A} \delta_{B}^{a} \delta_{c}^{C} x^{c}\right) \delta_{a}^{\beta^{\prime}} .
\end{gathered}
$$

To eliminate further excessive use of Kronecker deltas, let $p=3, D^{A}=\delta_{\alpha}^{A} D^{\alpha}$, and $d^{a}=\delta_{\alpha^{\prime}}^{a} d^{\alpha^{\prime}}$. Introducing a fundamental Finsler function $L(X, D)$ with properties 
described in Section $2.1(Y \rightarrow D)$, the following definitions hold [38]:

$$
\begin{gathered}
G_{A B}=\frac{1}{2} \frac{\partial^{2}\left(L^{2}\right)}{\partial D^{A} \partial D^{B}} ; \\
\gamma_{B C}^{A}=\frac{1}{2} G^{A D}\left(\frac{\partial G_{B D}}{\partial X^{C}}+\frac{\partial G_{C D}}{\partial X^{B}}-\frac{\partial G_{B C}}{\partial X^{D}}\right) ; \\
N_{B}^{A}=\frac{1}{2} \frac{\partial G^{A}}{\partial D^{B}}, \quad G^{A}=\gamma_{B C}^{A} D^{B} D^{C} ; \\
C_{A B C}=\frac{1}{2} \frac{\partial G_{A B}}{\partial D^{C}}=\frac{1}{4} \frac{\partial^{3}\left(L^{2}\right)}{\partial D^{A} \partial D^{B} \partial D^{C}} ; \\
\Gamma_{B C}^{A}=\frac{1}{2} G^{A D}\left(\frac{\delta G_{B D}}{\delta X^{C}}+\frac{\delta G_{C D}}{\delta X^{B}}-\frac{\delta G_{B C}}{\delta X^{D}}\right) ; \\
\frac{\delta}{\delta X^{A}}=\frac{\partial}{\partial X^{A}}-N_{A}^{B} \frac{\partial}{\partial D^{B}}, \quad \delta D^{A}=d D^{A}+N_{B}^{A} d X^{B} .
\end{gathered}
$$

Notice that $\Gamma_{B C}^{A}$ correspond to the Chern-Rund connection and $C_{A B C}$ to the Cartan tensor. In [36], D is presumed stationary $\left(B_{\beta}^{\alpha^{\prime}} \rightarrow \delta_{\beta}^{\alpha^{\prime}}\right)$ and is associated with a residual plastic disturbance, $C_{A B C}$ is remarked to be associated with dislocation density, and the HV-curvature tensor of $\Gamma_{B C}^{A}$ (e.g., $P_{B C D}^{A}$ of (19)) is remarked to be associated with disclination density. In [38], $\mathbf{B}$ is associated with lattice distortion and $L^{2}$ with residual strain energy density of the dislocation density $[6,56]$. In [40], a fundamental function and connection coefficients are not defined explicitly, leaving the theory open to generalization. Note also that (52) is more general than (45). When the latter holds and $\Gamma_{B C}^{A}=0$, then $\mathfrak{F} \rightarrow \mathbf{F}$, the usual deformation gradient of continuum mechanics.

Restricting attention to the time-independent case, a Lagrangian is posited of the form

$$
\begin{aligned}
\mathfrak{L} & (X, D) \\
& =\mathfrak{L}[X, D, x(X, D), \mathfrak{F}(X, D), \mathbf{Q}(X, D), \nabla \mathbf{Q}(X, D)],
\end{aligned}
$$

where $\mathbf{Q}$ is a generic vector of state variables and $\nabla$ denotes a generic gradient that may include partial, horizontal, and/or vertical covariant derivatives in the reference configuration as physically and mathematically appropriate. Let $\Omega$ be a compact domain of $Z$, and define

$$
\begin{aligned}
I(\Omega) & =\int_{\Omega} \widehat{\mathfrak{Q}}(X, D) d X^{1} \cdots \wedge d X^{n} \wedge d D^{1} \cdots \wedge d D^{p}, \\
\widehat{\mathfrak{L}} & =\left[\left|\operatorname{det}\left(G_{A B}\right)\right|\left|\operatorname{det}\left(\frac{\partial}{\partial X^{A}} \cdot \frac{\partial}{\partial X^{B}}\right)\right|\right]^{1 / 2} \mathfrak{L} .
\end{aligned}
$$

Euler-Lagrange equations referred to the reference configuration follow from the variational principle $\delta I=0$ analogously to (42). Conjugate forces to variations in kinematic and state variables can be defined as derivatives of $\mathbb{Q}$ with respect to these variables. Time dependence, dissipation, first and second laws of thermodynamics, and temperature effects are also considered in $[38,40]$; details are beyond the scope of this review. In the only known application of Finsler geometry to solve a boundary value problem in the context of mechanics of solids with microstructure, Stumpf and Saczuk [38] use the theory outlined above to study localization of plastic slip in a bar loaded in tension, with $D^{A}$ specifying a preferred material direction for slip. In [40], various choices of $\mathbf{Q}$ and its gradient are considered in particular energy functions, for example, state variables associated with gradients of damage parameters or void volume fractions.

\section{Towards a New Theory of Structured Media}

4.1. Background and Scope. In Section 4 it is shown how Finsler geometry can be applied to describe physical problems in deformable continua with evolving microstructures in a manner somewhat analogous to the phase field method. Phase field theory [57] encompasses various diffuse interface models wherein the boundary between two (or more) phases or states of material is distinguished by the gradient of a scalar field called an order parameter. The order parameter, denoted herein by $\eta$, typically varies continuously between values of zero and unity in phases one and two, with intermediate values in phase boundaries. Mathematically,

$$
\begin{aligned}
& \eta(X)=0 \quad \forall X \in \text { phase } 1, \\
& \eta(X)=1 \quad \forall X \in \text { phase } 2, \\
& \eta(X) \in(0,1) \quad \forall X \in \text { interface. }
\end{aligned}
$$

Physically, phases might correspond to liquid and solid in melting-solidification problems, austenite and martensite in structure transformations, vacuum and intact solid in fracture mechanics, or twin and parent crystal in twinning descriptions. Similarities between phase field theory and gradient-type theories of continuum mechanics are described in [58]; both classes of theory benefit from regularization associated with a length scale dependence of solutions that can render numerical solutions to governing equations mesh independent. Recent phase field theories incorporating finite deformation kinematics include [59] for martensitic transformations, [54] for fracture, [60] for amorphization, and [53] for twinning. The latter (i.e., deformation twinning) is the focus of a more specific description that follows later in Section 4.3 of this paper, though it is anticipated that the Finsler-type description could be adapted straightforwardly to describe other deformation physics.

Deformation twinning involves shearing and lattice rotation/reflection induced by mechanical stress in a solid crystal. The usual elastic driving force is a resolved shear stress on the habit plane, in the direction of twinning shear. Twinning can be reversible or irreversible depending on material and loading protocol; the physics of deformation twinning is described more fully in [61] and Chapter 8 of [6]. Theory that follows in Section 4.2 is thought to be the first to recognize 
analogies between phase field theory and Finsler geometry and that in Section 4.3 the first to apply Finsler geometric concepts to model deformation twinning. Notation follows that of Section 3.2 and Section 3.3 with possible exceptions highlighted as they appear.

4.2. Finsler Geometry and Kinematics. As in Section 3.3, define $\zeta=(Z, \pi, M, U)$ as a fiber bundle of total space $Z$ with $\pi: Z \rightarrow M$. Considered is a three-dimensional solid body with state vector $\mathbf{D}$ : dimensions of $M$ and $U$ are 3; the dimension of $Z$ is 6 . Coordinates on $Z$ are $\left\{X^{A}, D^{A}\right\}$, $(A=1,2,3)$, where $X \in M$ is a point on the base body in its reference configuration. Let $\zeta^{\prime}=\left(Z^{\prime}, \pi^{\prime}, M^{\prime}, U^{\prime}\right)$ be the deformed image of fiber bundle $\zeta$; dimensions of $M^{\prime}$ and $U^{\prime}$ are 3 , and that of $Z^{\prime}$ is 6 . Coordinates on $Z^{\prime}$ are $\left\{x^{a}, d^{a}\right\}$, where $x \in M^{\prime}$ is a point on the base body in its current configuration, and $\mathbf{d}$ is the updated state vector. Tangent bundles are expressed as direct sums of horizontal and vertical distributions as in (51):

$$
T Z=H Z \oplus V Z, \quad T Z^{\prime}=H Z^{\prime} \oplus V Z^{\prime} .
$$

Deformation of $Z$ to $Z^{\prime}$ is locally represented by the smooth and invertible coordinate transformations

$$
x^{a}=x^{a}\left(X^{A}, D^{A}\right), \quad d^{a}=B_{A}^{a} D^{A},
$$

where the director deformation function is, in general,

$$
B_{A}^{a}=B_{A}^{a}(X, D) .
$$

Introduce the smooth scalar order parameter field $\eta \in M$ as in (59), where $\eta=\eta(X)$. Here $\mathbf{D}$ is identified with the reference gradient of $\eta$ :

$$
D^{A}(X)=\delta^{A B} \frac{\partial \eta(X)}{\partial X^{B}}
$$

For simplicity, here $\left\{X^{A}\right\}$ is taken as a Cartesian coordinate chart on $M$ such that $X^{A}=\delta^{A B} X_{B}$, and so forth. Similarly, $\left\{x^{a}\right\}$ is chosen Cartesian. Generalization to curvilinear coordinates is straightforward but involves additional notation. Define the partial deformation gradient

$$
F_{A}^{a}(X, D)=\frac{\partial x^{a}(X, D)}{\partial X^{A}} .
$$

By definition and then from the chain rule,

$$
\begin{aligned}
d^{a}[x(X, D), D] & =\delta^{a b} \frac{\partial \eta(X)}{\partial x^{b}} \\
& =\delta^{a b}\left(\frac{\partial X^{A}}{\partial x^{b}}\right)\left(\frac{\partial \eta}{\partial X^{A}}\right) \\
& =F_{b}^{-1 A} \delta^{a b} \delta_{A B} D^{B}
\end{aligned}
$$

leading to

$$
B_{A}^{a}(X, D)=F_{b}^{-1 B}(X, D) \delta^{a b} \delta_{A B}
$$

From (63) and (65), local integrability (null curl) conditions $\partial D^{A} / \partial X^{B}=\partial D^{B} / \partial X^{A}$ and $\partial d^{a} / \partial x^{b}=\partial d^{b} / \partial x^{a}$ hold. As in Section $3, N_{B}^{A}(X, D)$ denote nonlinear connection coefficients on $Z$, and the Finsler-type nonholonomic bases

$$
\frac{\delta}{\delta X^{A}}=\frac{\partial}{\partial X^{A}}-N_{A}^{B} \frac{\partial}{\partial D^{B}}, \quad \delta D^{A}=d D^{A}+N_{B}^{A} d X^{B}
$$

Let $N_{b}^{a}=N_{B}^{A} \delta_{A}^{a} \delta_{b}^{B}$ denote nonlinear connection coefficients on $Z^{\prime}$ [38], and define the nonholonomic spatial bases as

$$
\frac{\delta}{\delta x^{a}}=\frac{\partial}{\partial x^{a}}-N_{a}^{b} \frac{\partial}{\partial d^{b}}, \quad \delta d^{a}=d d^{a}+N_{b}^{a} d x^{b}
$$

Total deformation gradient $\mathfrak{F}: T Z \rightarrow T Z^{\prime}$ is defined as

$$
\mathfrak{F}=\overline{\mathbf{F}}+\widehat{\mathbf{F}}=x_{\mid A}^{a} \frac{\delta}{\delta x^{a}} \otimes d X^{A}+x_{\| A}^{a} \frac{\partial}{\partial d^{a}} \otimes \delta D^{A} .
$$

In component form, its horizontal and vertical parts are, as in (55) and the theory of [38],

$$
\begin{aligned}
& \bar{F}_{A}^{a}=x_{\mid A}^{a}=\frac{\delta x^{a}}{\delta X^{A}}+\Gamma_{A C}^{B} \delta_{B}^{a} \delta_{c}^{C} x^{c}, \\
& \widehat{F}_{A}^{a}=x_{\| A}^{a}=\frac{\partial x^{a}}{\partial D^{A}}+C_{A C}^{B} \delta_{B}^{a} \delta_{c}^{C} x^{c} .
\end{aligned}
$$

As in Section 3.3, a fundamental Finsler function $L(X, D)$, homogeneous of degree one in $D$, is introduced. Then

$$
\begin{gathered}
G_{A B}=\frac{1}{2} \frac{\partial^{2}\left(L^{2}\right)}{\partial D^{A} \partial D^{B}} ; \\
\gamma_{B C}^{A}=\frac{1}{2} G^{A D}\left(\frac{\partial G_{B D}}{\partial X^{C}}+\frac{\partial G_{C D}}{\partial X^{B}}-\frac{\partial G_{B C}}{\partial X^{D}}\right) ; \\
N_{B}^{A}=\frac{1}{2} \frac{\partial G^{A}}{\partial D^{B}}, \quad G^{A}=\gamma_{B C}^{A} D^{B} D^{C} ; \\
C_{A B C}=\frac{1}{2} \frac{\partial G_{A B}}{\partial D^{C}}=\frac{1}{4} \frac{\partial^{3}\left(L^{2}\right)}{\partial D^{A} \partial D^{B} \partial D^{C}} \\
\Gamma_{B C}^{A}=\frac{1}{2} G^{A D}\left(\frac{\delta G_{B D}}{\delta X^{C}}+\frac{\delta G_{C D}}{\delta X^{B}}-\frac{\delta G_{B C}}{\delta X^{D}}\right) .
\end{gathered}
$$

Specifically for application of the theory in the context of initially homogeneous single crystals, let

$$
\begin{gathered}
L=\left[\kappa_{A B} D^{A} D^{B}\right]^{1 / 2}=\left[\kappa^{A B}\left(\frac{\partial \eta}{\partial X^{A}}\right)\left(\frac{\partial \eta}{\partial X^{B}}\right)\right]^{1 / 2}, \\
\kappa_{A B}=G_{A B}=\delta_{A C} \delta_{B D} G^{C D}=\text { constant. }
\end{gathered}
$$

The metric tensor with Cartesian components $\kappa_{A B}$ is identified with the gradient energy contribution to the surface energy term in phase field theory [53], as will be made explicit later in Section 4.3. From (76) and (77), reference geometry $\zeta$ is now Minkowskian since the fundamental Finsler function 
$L=L(D)$ is independent of $X$. In this case, (72)-(75) and (67)-(68) reduce to

$$
\begin{array}{cc}
\Gamma_{B C}^{A}=\gamma_{B C}^{A}=C_{B C}^{A}=0 ; & N_{B}^{A}=0, \quad n_{b}^{a}=0 ; \\
\frac{\delta}{\delta X^{A}}=\frac{\partial}{\partial X^{A}}, & \delta D^{A}=d D^{A} ; \\
\frac{\delta}{\delta x^{a}}=\frac{\partial}{\partial x^{a}}, & \delta d^{a}=d d^{a} .
\end{array}
$$

Horizontal and vertical covariant derivatives in (69) reduce to partial derivatives with respect to $X^{A}$ and $D^{A}$, respectively, leading to

$$
\begin{aligned}
\mathfrak{F} & =\frac{\partial x^{a}}{\partial X^{A}} \frac{\partial}{\partial x^{a}} \otimes d X^{A}+\frac{\partial x^{a}}{\partial D^{A}} \frac{\partial}{\partial d^{a}} \otimes d D^{A} \\
& =F_{A}^{a} \frac{\partial}{\partial x^{a}} \otimes d X^{A}+V_{A}^{a} \frac{\partial}{\partial d^{a}} \otimes d D^{A} .
\end{aligned}
$$

When $x=x(X)$, then vertical deformation components $\widehat{F}_{A}^{a}=V_{A}^{a}=0$. In a more general version of (76) applicable to heterogeneous material properties, $\kappa_{A B}=\kappa_{A B}(X, D)$ and is homogeneous of degree zero with respect to $D$, and the above simplifications (i.e., vanishing connection coefficients) need not apply.

4.3. Governing Equations: Twinning Application. Consider a crystal with a single potentially active twin system. Applying (59), let $\eta(X)=1 \forall X \in$ twinned domains, $\eta(X)=0 \forall X \in$ parent (original) crystal domains, and $\eta(X) \in(0,1) \forall X \in$ in twin boundary domains. As defined in [53], let $\gamma_{0}$ denote the twinning eigenshear (a scalar constant), $\mathbf{m}=m_{A} d X^{A}$ denote the unit normal to the habit plane (i.e., the normal covector to the twin boundary), and $\mathbf{s}=s^{A}\left(\partial / \partial X^{A}\right)$ the direction of twinning shear. In the context of the geometric framework of Section 4.2, and with simplifying assumptions (76)-(79) applied throughout the present application, $s \in T M$ and $\mathbf{m} \in T^{*} M$ are constant fields that obey the orthonormality conditions

$$
\langle\mathbf{s}, \mathbf{m}\rangle=s^{A} m_{B}\left\langle\frac{\partial}{\partial X^{A}}, d X^{B}\right\rangle=s^{A} m_{A}=0 .
$$

Twinning deformation is defined as the $\left(\begin{array}{l}1 \\ 1\end{array}\right)$ tensor field

$$
\begin{aligned}
& \boldsymbol{\Xi}=\Xi_{B}^{A} \frac{\partial}{\partial X^{A}} \otimes d X^{B}=\mathbf{1}+\gamma_{0} \varphi \mathbf{s} \otimes \mathbf{m}, \\
& \Xi_{B}^{A}[\eta(X)]=\delta_{B}^{A}+\gamma_{0} \varphi[\eta(X)] s^{A} m_{B} .
\end{aligned}
$$

Note that $\operatorname{det} \boldsymbol{\Xi}=1+\gamma_{0} \varphi\langle\mathbf{s}, \mathbf{m}\rangle=1$. Scalar interpolation function $\varphi(\eta) \in[0,1]$ monotonically increases between its endpoints with increasing $\eta$, and it satisfies $\varphi(0)=0, \varphi(1)=$ 1 , and $\varphi^{\prime}(0)=\varphi^{\prime}(1)=0$. A typical example is the cubic polynomial $[53,59]$

$$
\varphi[\eta(X)]=3[\eta(X)]^{2}-2[\eta(X)]^{3} .
$$

The horizontal part of the deformation gradient in (79) obeys a multiplicative decomposition:

$$
\mathbf{F}=\mathbf{A} \Xi, \quad F_{A}^{a}=A_{B}^{a} \Xi_{A}^{B} .
$$

The elastic lattice deformation is the two-point tensor A:

$$
\mathbf{A}=A_{A}^{a} \frac{\partial}{\partial x^{a}} \otimes d X^{B}=\mathbf{F} \mathbf{\Xi}^{-1}=\mathbf{F}\left(\mathbf{1}-\gamma_{0} \varphi \mathbf{s} \otimes \mathbf{m}\right) .
$$

Note that (83) can be considered a version of the BilbyKröner decomposition proposed for elastic-plastic solids [8, $62]$ and analyzed at length in $[5,11,26]$ from perspectives of differential geometry of anholonomic space (neither $\mathbf{A}$ nor $\boldsymbol{\Xi}$ is necessarily integrable to a vector field). The following energy potentials, measured per unit reference volume, are defined:

$$
\begin{gathered}
\psi(\mathfrak{F}, \eta, \mathbf{D})=W(\mathfrak{F}, \eta)+f(\eta, \mathbf{D}) ; \\
W=W[\mathbf{A}(\mathbf{F}, \eta)], \quad f(\eta, \mathbf{D})=\alpha \eta^{2}(1-\eta)^{2}+L^{2}(\mathbf{D}) .
\end{gathered}
$$

Here, $W$ is the strain energy density that depends on elastic lattice deformation A (assuming $V_{A}^{a}=x_{\| A}^{a}=0$ in (86)), $f$ is the total interfacial energy that includes a double-well function with constant coefficient $\alpha$, and $L^{2}=\kappa_{A B} D^{A} D^{B}$ is the square of the fundamental Finsler function given in (76). For isotropic twin boundary energy $\kappa_{A B}=\kappa_{0} \delta_{A B}$, and equilibrium surface energy $\Gamma_{0}$ and regularization width $l_{0}$ obey $\kappa_{0}=(3 / 4) \Gamma_{0} l_{0}$ and $\alpha=12 \Gamma_{0} / l_{0}$ [53]. The total energy potential per unit volume is $\psi$. Let $\Omega$ be a compact domain of $Z$, which can be identified as a region of the material body, and define the total potential energy functional

$$
\begin{gathered}
\Psi[x(X), \eta(X)]=\int_{\Omega} \psi \mathrm{d} \Omega, \\
\mathrm{d} \Omega=\left|\operatorname{det}\left(G_{A B}\right)\right|^{1 / 2} d X^{1} \cdots \wedge d X^{n} \wedge d D^{1} \cdots \wedge d D^{p} .
\end{gathered}
$$

Recall that for solid bodies, $n=p=3$. For quasi-static conditions (null kinetic energy), the Lagrangian energy density is $\mathbf{Q}=-\psi$. The null first variation of the action integral, appropriate for essential (Dirichlet) boundary conditions on boundary $\partial \Omega$, is

$$
\delta I(\Omega)=-\delta \Psi=-\int_{\Omega} \delta \psi \mathrm{d} \Omega=0,
$$

where the first variation of potential energy density is defined here by varying $x$ and $\eta$ within $\Omega$, holding reference coordinates $X$ and reference volume form $\mathrm{d} \Omega$ fixed:

$$
\delta \psi=\frac{\partial W}{\partial F_{A}^{a}} \frac{\partial \delta x^{a}}{\partial X^{A}}+\left(\frac{\partial W}{\partial \eta}+\frac{\partial f}{\partial \eta}\right) \delta \eta+\frac{\partial f}{\partial D^{A}} \frac{\partial \delta \eta}{\partial X^{A}} .
$$

Application of the divergence theorem, here with vanishing variations of $x$ and $\eta$ on $\partial \Omega$, leads to the Euler-Lagrange equations [53]

$$
\begin{gathered}
\frac{\partial P_{a}^{A}}{\partial X^{A}}=\frac{\partial\left(\partial W / \partial x_{\mid A}^{a}\right)}{\partial X^{A}}=0 \\
\varsigma+\frac{\partial f}{\partial \eta}=2 \kappa_{A B} \frac{\partial D^{A}}{\partial X_{B}}=2 \kappa_{A B} \frac{\partial^{2} \eta}{\partial X_{A} \partial X_{B}} .
\end{gathered}
$$


The first Piola-Kirchhoff stress tensor is $\mathbf{P}=\partial W / \partial \mathbf{F}$, and the elastic driving force for twinning is $\varsigma=\partial W / \partial \eta$. These equations, which specify mechanical and phase equilibria, are identical to those derived in [53], but have arrived here via use of Finsler geometry on fiber bundle $\zeta$. In order to achieve such correspondence, simplifications $L(X, D) \rightarrow L(D)$ and $x(X, D) \rightarrow x(X)$ have been applied, the first reducing the fundamental Finsler function to one of Minkowskian geometry to describe energetics of twinning in an initially homogeneous single crystal body $\left(\kappa_{A B}=\right.$ constant).

A more general and potentially powerful approach would be to generalize fundamental function $L$ and deformed coordinates $x$ to allow for all possible degrees-of-freedom. For example, a fundamental function $L(X, D)$ corresponding to nonuniform values of $\kappa_{A B}(X)$ in the vicinity of grain or phase boundaries, wherein properties change rapidly with position $X$, could be used instead of a Minkowskian (position-independent) fundamental function $L(D)$. Such generalization would lead to enriched kinematics and nonvanishing connection coefficients, and it may yield new physical and mathematical insight into equilibrium equations-for example, when expressed in terms of horizontal and vertical covariant derivatives [30] - used to describe mechanics of interfaces and heterogeneities such as inclusions or other defects. Further study, to be pursued in the future, is needed to relate such a general geometric description to physical processes in real heterogeneous materials. An analogous theoretical description could be derived straightforwardly to describe stress-induced amorphization or cleavage fracture in crystalline solids, extending existing phase field models $[54,60]$ of such phenomena.

\section{Conclusions}

Finsler geometry and its prior applications towards continuum physics of materials with microstructure have been reviewed. A new theory, in general considering a deformable vector bundle of Finsler character, has been posited, wherein the director vector of Finsler space is associated with a gradient of a scalar order parameter. It has been shown how a particular version of the new theory (Minkowskian geometry) can reproduce governing equations for phase field modeling of twinning in initially homogeneous single crystals. A more general approach allowing the fundamental function to depend explicitly on material coordinates has been posited that would offer enriched description of interfacial mechanics in polycrystals or materials with multiple phases.

\section{Conflict of Interests}

The author declares that there is no conflict of interests regarding the publication of this paper.

\section{References}

[1] C. A. Truesdell and R. A. Toupin, "The classical field theories," in Handbuch der Physik, S. Flugge, Ed., vol. 3/1, pp. 226-793, Springer, Berlin, Germany, 1960.
[2] A. C. Eringen, Nonlinear Theory of Continuous Media, McGrawHill, New York, NY, USA, 1962.

[3] A. C. Eringen, "Tensor analysis," in Continuum Physics, A. C. Eringen, Ed., vol. 1, pp. 1-155, Academic Press, New York, NY, USA, 1971.

[4] R. A. Toupin, "Theories of elasticity with couple-stress," Archive for Rational Mechanics and Analysis, vol. 17, pp. 85-112, 1964.

[5] J. D. Clayton, "On anholonomic deformation, geometry, and differentiation," Mathematics and Mechanics of Solids, vol. 17, no. 7, pp. 702-735, 2012.

[6] J. D. Clayton, Nonlinear Mechanics of Crystals, Springer, Dordrecht, The Netherlands, 2011.

[7] B. A. Bilby, R. Bullough, and E. Smith, "Continuous distributions of dislocations: a new application of the methods of nonRiemannian geometry," Proceedings of the Royal Society A, vol. 231, pp. 263-273, 1955.

[8] E. Kröner, "Allgemeine kontinuumstheorie der versetzungen und eigenspannungen," Archive for Rational Mechanics and Analysis, vol. 4, pp. 273-334, 1960.

[9] K. Kondo, "On the analytical and physical foundations of the theory of dislocations and yielding by the differential geometry of continua," International Journal of Engineering Science, vol. 2, pp. 219-251, 1964.

[10] B. A. Bilby, L. R. T. Gardner, A. Grinberg, and M. Zorawski, "Continuous distributions of dislocations. VI. Non-metric connexions," Proceedings of the Royal Society of London A: Mathematical, Physical and Engineering Sciences, vol. 292, no. 1428, pp. 105-121, 1966.

[11] W. Noll, "Materially uniform simple bodies with inhomogeneities," Archive for Rational Mechanics and Analysis, vol. 27, no. 1, pp. 1-32, 1967.

[12] K. Kondo, "Fundamentals of the theory of yielding elementary and more intrinsic expositions: riemannian and nonriemannian terminology," Matrix and Tensor Quarterly, vol. 34, pp. 55-63, 1984.

[13] J. D. Clayton, D. J. Bammann, and D. L. McDowell, "A geometric framework for the kinematics of crystals with defects," Philosophical Magazine, vol. 85, no. 33-35, pp. 3983-4010, 2005.

[14] J. D. Clayton, "Defects in nonlinear elastic crystals: differential geometry, finite kinematics, and second-order analytical solutions," Zeitschrift für Angewandte Mathematik und Mechanik, 2013.

[15] A. Yavari and A. Goriely, "The geometry of discombinations and its applications to semi-inverse problems in anelasticity," Proceedings of the Royal Society of London A, vol. 470, article 0403, 2014.

[16] D. G. B. Edelen and D. C. Lagoudas, Gauge Theory and Defects in Solids, North-Holland Publishing, Amsterdam, The Netherlands, 1988.

[17] I. A. Kunin, "Kinematics of media with continuously changing topology," International Journal of Theoretical Physics, vol. 29, no. 11, pp. 1167-1176, 1990.

[18] H. Weyl, Space-Time-Matter, Dover, New York, NY, USA, 4th edition, 1952.

[19] J. A. Schouten, Ricci Calculus, Springer, Berlin, Germany, 1954.

[20] J. L. Ericksen, "Tensor Fields," in Handbuch der Physik, S. Flugge, Ed., vol. 3, pp. 794-858, Springer, Berlin, Germany, 1960.

[21] T. Y. Thomas, Tensor Analysis and Differential Geometry, Academic Press, New York, NY, USA, 2nd edition, 1965.

[22] M. A. Grinfeld, Thermodynamic Methods in the Theory of Heterogeneous Systems, Longman, Sussex, UK, 1991. 
[23] H. Stumpf and U. Hoppe, "The application of tensor algebra on manifolds to nonlinear continuum mechanics-invited survey article," Zeitschrift für Angewandte Mathematik und Mechanik, vol. 77, no. 5, pp. 327-339, 1997.

[24] P. Grinfeld, Introduction to Tensor Analysis and the Calculus of Moving Surfaces, Springer, New York, NY, USA, 2013.

[25] P. Steinmann, "On the roots of continuum mechanics in differential geometry-a review," in Generalized Continua from the Theory to Engineering Applications, H. Altenbach and V. A. Eremeyev, Eds., vol. 541 of CISM International Centre for Mechanical Sciences, pp. 1-64, Springer, Udine, Italy, 2013.

[26] J. D. Clayton, Differential Geometry and Kinematics of Continua, World Scientific, Singapore, 2014.

[27] V. A. Eremeyev, L. P. Lebedev, and H. Altenbach, Foundations of Micropolar Mechanics, Springer Briefs in Applied Sciences and Technology, Springer, Heidelberg, Germany, 2013.

[28] P. Finsler, Über Kurven und Flächen in allgemeinen Räumen [Dissertation], Gottingen, Germany, 1918.

[29] H. Rund, The Differential Geometry of Finsler Spaces, Springer, Berlin, Germany, 1959.

[30] A. Bejancu, Finsler Geometry and Applications, Ellis Horwood, New York, NY, USA, 1990.

[31] D. Bao, S.-S. Chern, and Z. Shen, An Introduction to RiemannFinsler Geometry, Springer, New York, NY, USA, 2000.

[32] A. Bejancu and H. R. Farran, Geometry of Pseudo-Finsler Submanifolds, Kluwer Academic Publishers, Dordrecht, The Netherlands, 2000.

[33] E. Kröner, "Interrelations between various branches of continuum mechanics," in Mechanics of Generalized Continua, pp. 330-340, Springer, Berlin, Germany, 1968.

[34] E. Cartan, Les Espaces de Finsler, Hermann, Paris, France, 1934.

[35] S. Ikeda, "A physico-geometrical consideration on the theory of directors in the continuum mechanics of oriented media," The Tensor Society, Tensor: New Series, vol. 27, pp. 361-368, 1973.

[36] J. Saczuk, "On the role of the Finsler geometry in the theory of elasto-plasticity," Reports on Mathematical Physics, vol. 39, no. 1, pp. 1-17, 1997.

[37] M. F. Fu, J. Saczuk, and H. Stumpf, "On fibre bundle approach to a damage analysis," International Journal of Engineering Science, vol. 36, no. 15, pp. 1741-1762, 1998.

[38] H. Stumpf and J. Saczuk, "A generalized model of oriented continuum with defects," Zeitschrift für Angewandte Mathematik und Mechanik, vol. 80, no. 3, pp. 147-169, 2000.

[39] J. Saczuk, "Continua with microstructure modelled by the geometry of higher-order contact," International Journal of Solids and Structures, vol. 38, no. 6-7, pp. 1019-1044, 2001.

[40] J. Saczuk, K. Hackl, and H. Stumpf, "Rate theory of nonlocal gradient damage-gradient viscoinelasticity," International Journal of Plasticity, vol. 19, no. 5, pp. 675-706, 2003.

[41] S. Ikeda, "On the theory of fields in Finsler spaces," Journal of Mathematical Physics, vol. 22, no. 6, pp. 1215-1218, 1981.

[42] H. E. Brandt, "Differential geometry of spacetime tangent bundle," International Journal of Theoretical Physics, vol. 31, no. 3, pp. 575-580, 1992.

[43] I. Suhendro, "A new Finslerian unified field theory of physical interactions," Progress in Physics, vol. 4, pp. 81-90, 2009.

[44] S.-S. Chern, "Local equivalence and Euclidean connections in Finsler spaces," Scientific Reports of National Tsing Hua University Series A, vol. 5, pp. 95-121, 1948.
[45] K. Yano and E. T. Davies, "On the connection in Finsler space as an induced connection," Rendiconti del Circolo Matematico di Palermo, Serie II, vol. 3, pp. 409-417, 1954.

[46] A. Deicke, "Finsler spaces as non-holonomic subspaces of Riemannian spaces," Journal of the London Mathematical Society, vol. 30, pp. 53-58, 1955.

[47] S. Ikeda, "A geometrical construction of the physical interaction field and its application to the rheological deformation field," Tensor, vol. 24, pp. 60-68, 1972.

[48] Y. Takano, “Theory of fields in Finsler spaces. I," Progress of Theoretical Physics, vol. 40, pp. 1159-1180, 1968.

[49] J. D. Clayton, D. L. McDowell, and D. J. Bammann, "Modeling dislocations and disclinations with finite micropolar elastoplasticity," International Journal of Plasticity, vol. 22, no. 2, pp. 210256, 2006.

[50] T. Hasebe, "Interaction fields based on incompatibility tensor in field theory of plasticity-part I: theory," Interaction and Multiscale Mechanics, vol. 2, no. 1, pp. 1-14, 2009.

[51] J. D. Clayton, "Dynamic plasticity and fracture in high density polycrystals: constitutive modeling and numerical simulation," Journal of the Mechanics and Physics of Solids, vol. 53, no. 2, pp. 261-301, 2005.

[52] J. R. Mayeur, D. L. McDowell, and D. J. Bammann, "Dislocationbased micropolar single crystal plasticity: comparison of multiand single criterion theories," Journal of the Mechanics and Physics of Solids, vol. 59, no. 2, pp. 398-422, 2011.

[53] J. D. Clayton and J. Knap, "A phase field model of deformation twinning: nonlinear theory and numerical simulations," Physica D. Nonlinear Phenomena, vol. 240, no. 9-10, pp. 841-858, 2011.

[54] J. D. Clayton and J. Knap, "A geometrically nonlinear phase field theory of brittle fracture," International Journal of Fracture, vol. 189, no. 2, pp. 139-148, 2014.

[55] J. D. Clayton and D. L. McDowell, "A multiscale multiplicative decomposition for elastoplasticity of polycrystals," International Journal of Plasticity, vol. 19, no. 9, pp. 1401-1444, 2003.

[56] J. D. Clayton, "An alternative three-term decomposition for single crystal deformation motivated by non-linear elastic dislocation solutions," The Quarterly Journal of Mechanics and Applied Mathematics, vol. 67, no. 1, pp. 127-158, 2014.

[57] H. Emmerich, The Diffuse Interface Approach in Materials Science: Thermodynamic Concepts and Applications of PhaseField Models, Springer, Berlin, Germany, 2003.

[58] G. Z. Voyiadjis and N. Mozaffari, "Nonlocal damage model using the phase field method: theory and applications," International Journal of Solids and Structures, vol. 50, no. 20-21, pp. 3136-3151, 2013.

[59] V. I. Levitas, V. A. Levin, K. M. Zingerman, and E. I. Freiman, "Displacive phase transitions at large strains: phase-field theory and simulations," Physical Review Letters, vol. 103, no. 2, Article ID 025702, 2009.

[60] J. D. Clayton, "Phase field theory and analysis of pressure-shear induced amorphization and failure in boron carbide ceramic," AIMS Materials Science, vol. 1, no. 3, pp. 143-158, 2014.

[61] V. S. Boiko, R. I. Garber, and A. M. Kosevich, Reversible Crystal Plasticity, AIP Press, New York, NY, USA, 1994.

[62] B. A. Bilby, L. R. T. Gardner, and A. N. Stroh, "Continuous distributions of dislocations and the theory of plasticity," in Proceedings of the 9th International Congress of Applied Mechanics, vol. 8, pp. 35-44, University de Bruxelles, Brussels, Belgium, 1957. 


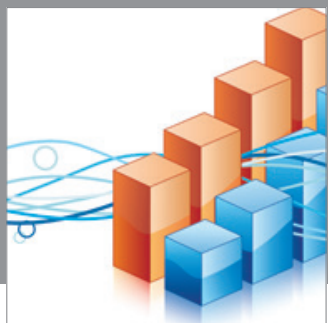

Advances in

Operations Research

mansans

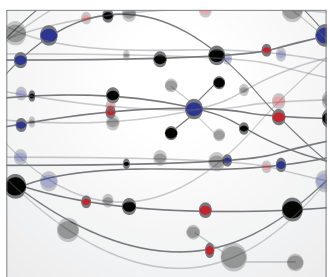

The Scientific World Journal
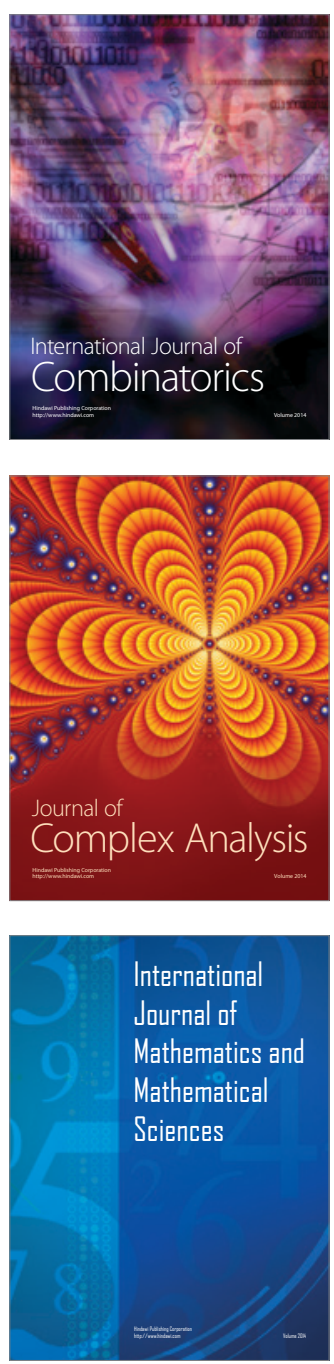
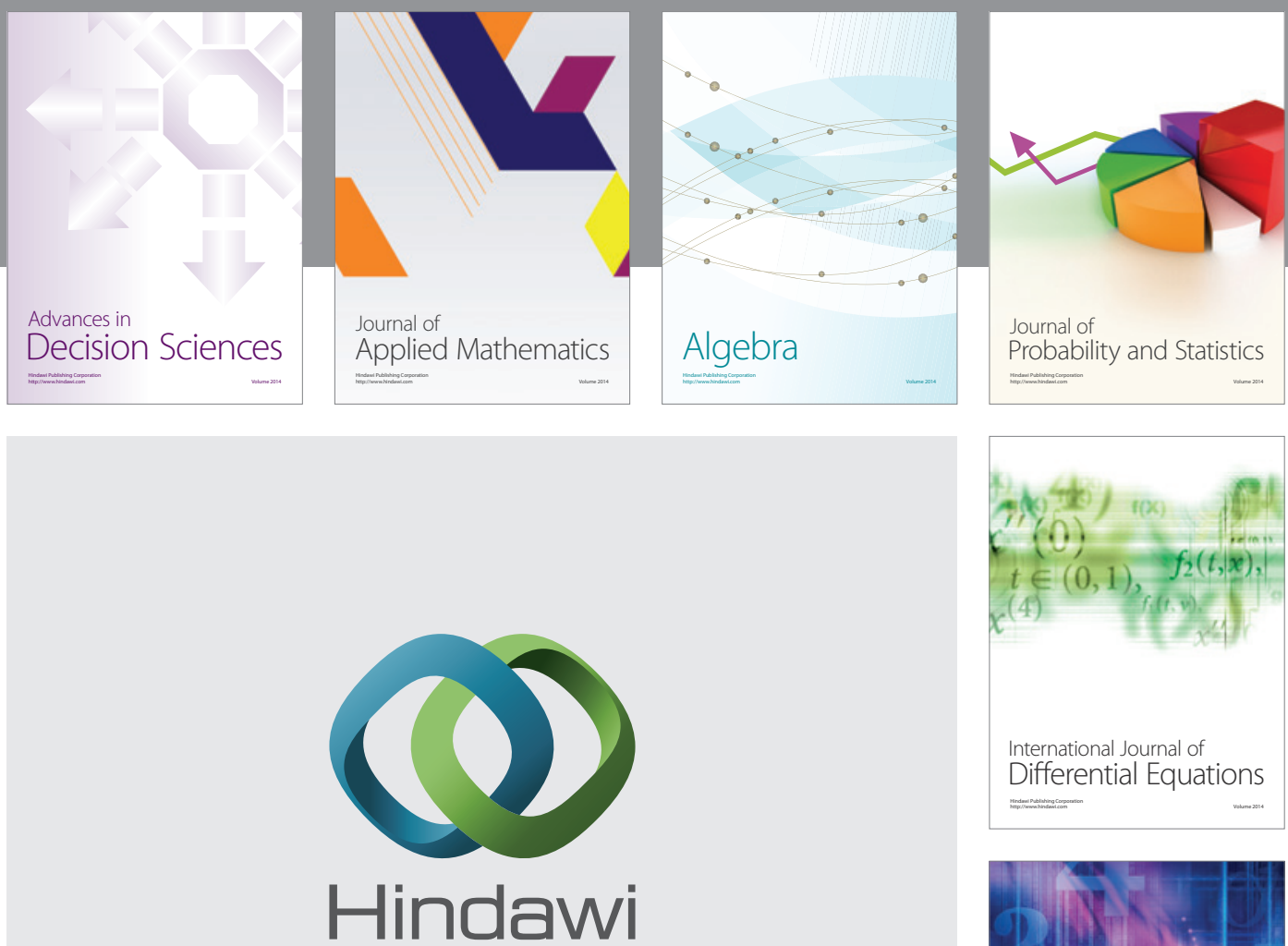

Submit your manuscripts at http://www.hindawi.com
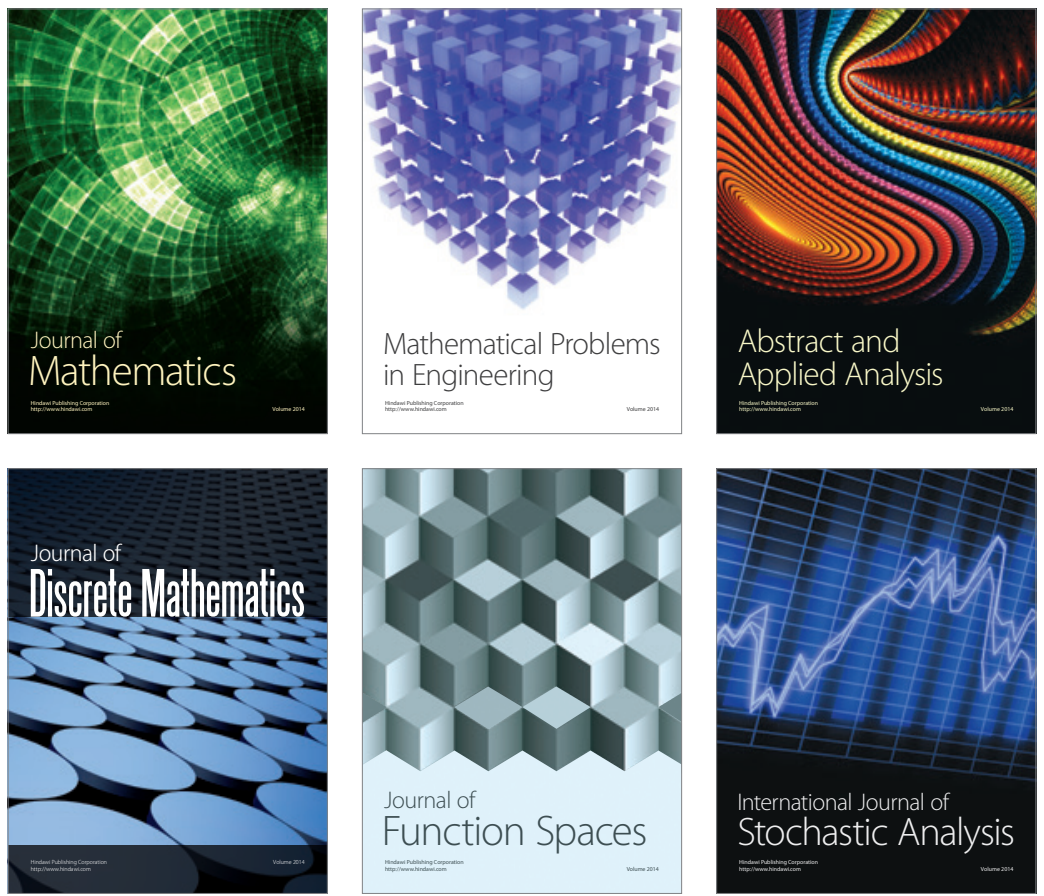

Journal of

Function Spaces

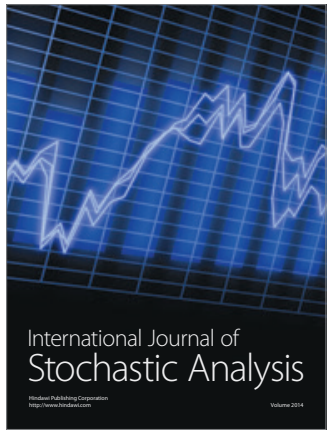

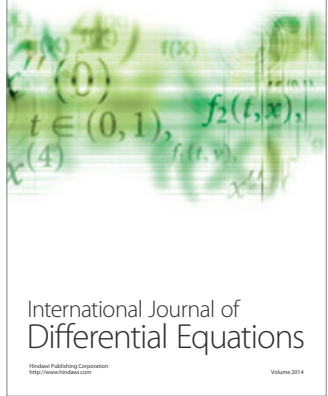
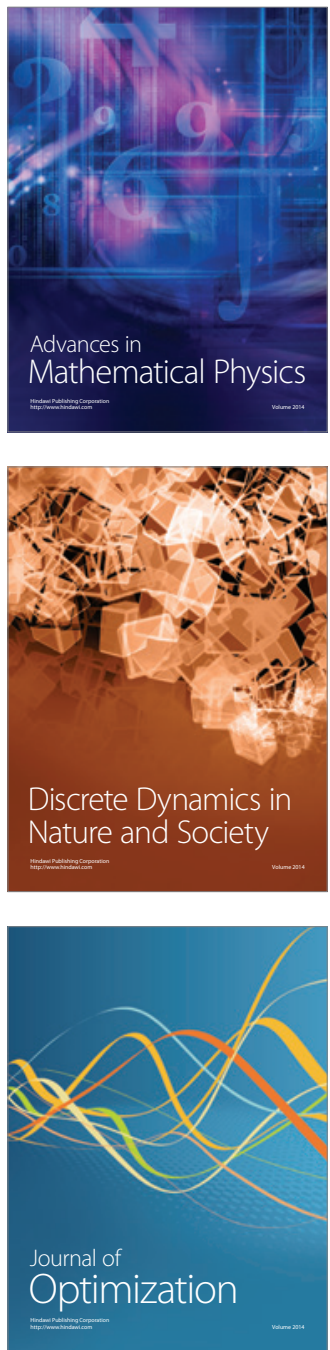